\title{
Verification of the method of equivalent amplitude determination based on two parameter fatigue characteristic
}

\author{
Karolina Karolewska ${ }^{1, *}$, Bogdan Ligaj ${ }^{1}$ \\ ${ }^{1}$ UTP University of Science and Technology in Bydgoszcz, Faculty of Mechanical Engineering, al. \\ Prof. S. Kaliskiego 7, 85-796 Bydgoszcz, Poland
}

\begin{abstract}
In the most causes the loads which are affected on structural components are various over time and their character changes is stochastic. The stochastic character of operational loads of construction elements in various machine types is depended on many factors, included : work forces variability, environmental conditions, physical properties of components etc. Fatigue life calculation for this type of loads are conducted on the basis of determined sinusoidal cycles set through to use of the cycles counting method. The cycles which are contained to the sinusoidal cycles set are characterized by extensive range of amplitude $S_{a i}$ variation and mean values $\mathrm{S}_{\mathrm{mi}}$. Application of $\mathrm{S}_{\mathrm{a}} \mathrm{N}$ curve in fatigue life calculations caused disregard of the cycle mean value. This may affect the accuracy of calculations. Taking into account the cycle mean value $S_{m}$ in the calculations may be realized by determining a substitute cycle with an average value $S_{m}=0$ and a substitute amplitude $S_{a z} \neq S_{a}$.
\end{abstract}

\section{Abbreviations}

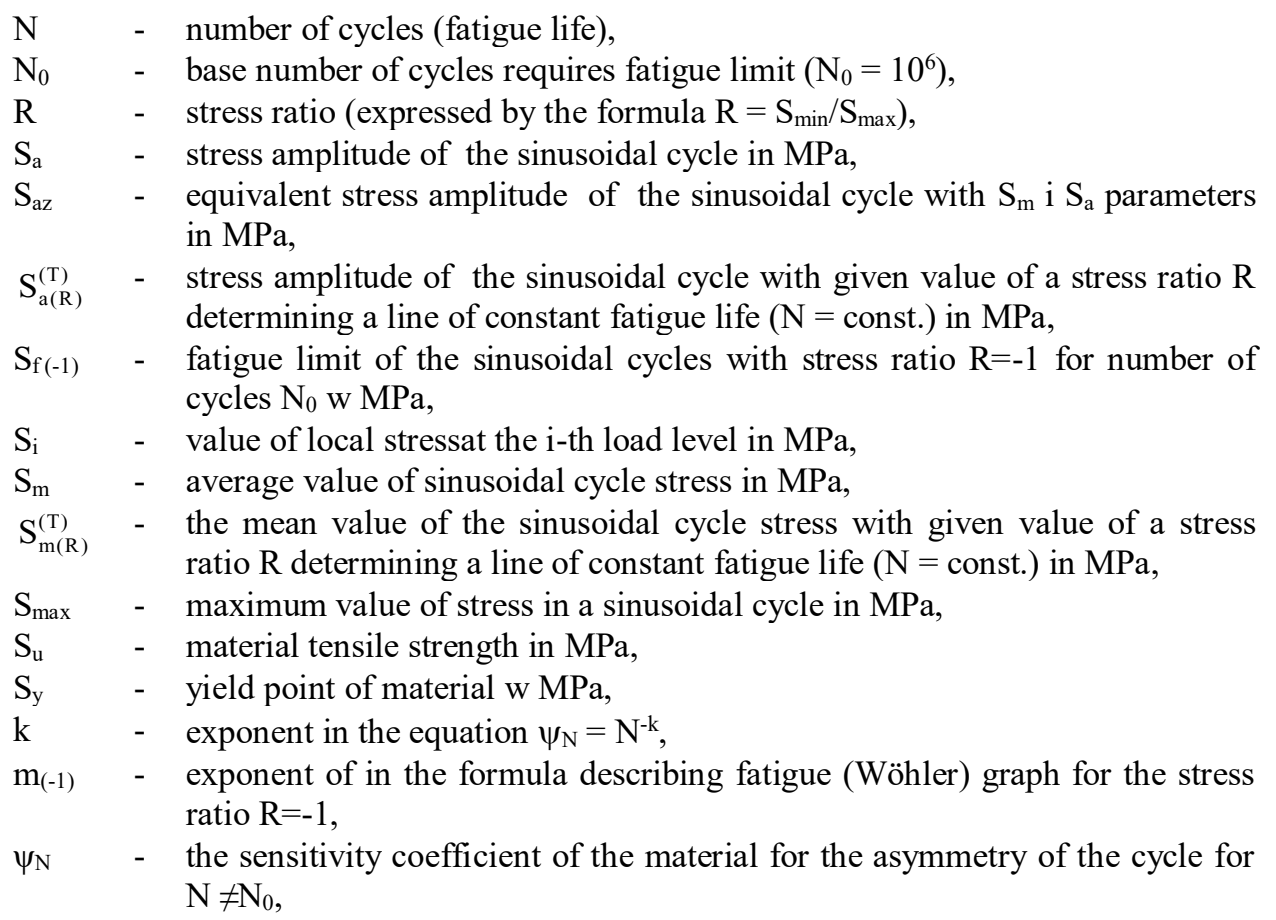

\footnotetext{
* Corresponding author: karolina.karolewska@utp.edu.pl
} 
$\lambda \quad-$ number of repetitions of a program to fatigue fracture.

\section{Introduction}

Fatigue life assessment is one of the main criteria during the design process of devices elements. On the basis of the fatigue life calculations under random loads conditions the design features selection of machine elements are made. Fatigue life determination for operational loads requires the use of characteristic describing the material properties, cumulative damage model and load spectrum developed for indicated loads.

Operational loads can be divided into two groups: narrow or broad spectrum. Due to fatigue life between the mentioned loads significant differences refer to cycles participation from a given range of the stress ratio $\mathrm{R}$ values and relate to occurrence of cycles with large amplitude values, where the maximum amplitude $\mathrm{S}_{\mathrm{a}} \approx \mathrm{S}_{\max }$.

Calculations of fatigue life for load chracterized by narrow spectrum are carried out by use of block load spectra (the spectra 1D) developed on the basis of determined cycles set by using one of the cycles counting method. The realization of fatigue life calculations in cause of board spectrum loads is based on loads specta describing variability of cycles parameters $S_{m}$ and $S_{a i}$ (the spectra 2D). The use of this type of spectrum is connected with cycles stress ratio values $-\infty<\mathrm{R}<+\infty$ occuarncy in cycles loads and their impact on fatigue life [1].

Replacement of load two-parameter spectrum (the spectra 2D) by one-parametre spectrum (the specta 1D) is based on determination substitite amplitude method $\mathrm{S}_{\mathrm{az}}$ with the use of two-parameter fatigue charakteristic [2]. For the purposes of the paper two-parameter characteristics denoted as : model IM (Fig. 2a), and model II (Fig.2b) were estabilished.

The aim of the paper is to compare determining fatigue life methods with the use of load spectrum. The load spectra were obtained using one of the cycles counting method, which were full cycles counting method, and also by using two-parameter characteristic: the IM model and II model.

The scope of the paper contains the presentation of determination substitute amplitude methods, comparison of fatigue life calculation results with the experimental test results for indicated operational loads.

\section{The method of determining substitute cycles of load}

Calculations of fatigue life under operational loads require replacing a random cycles load (Fig1.a) with sinusoidal cycles. To determine load equivalent cycles, one of the cycles counting method is used: peak counting method, simple-range counting method, full cycles counting method, range-pair counting method and rainflow counting method [4].

By means of one of the cycles counting method, sinusoidal cycles are obtained. The recived cycles vary in the stress amplitude values $S_{a i}$ and mean values $S_{m i}$ (Fig. 1b). Sets of cycles with different amplitude values $S_{a i}$ and mean value $S_{m i}$ are replaced by equivalent cycles with a mean value $S_{m i}=0$ and equivalent amplitude $S_{a z i}$ on the basis of two-parameter characteristics.

a)

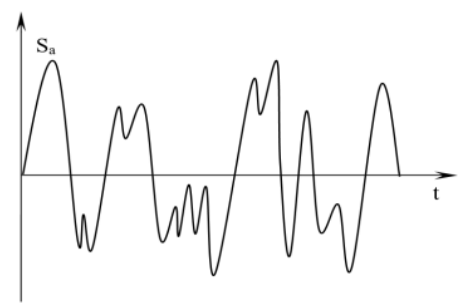

b)

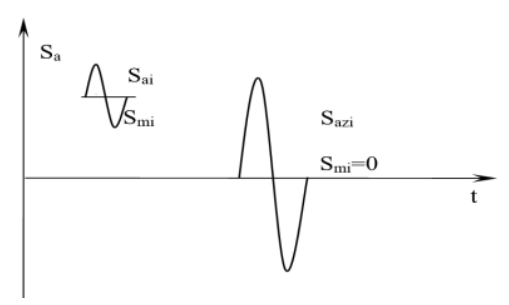

Fig. 1. Schematic presentation of loads: a) random course of load, b) selected load cycles. 
Two-parameter fatigue characteristics are used to determine equivalent $S_{\text {azi }}$ amplitudes. In this paper, the IM model (Fig.2a) and II model (Fig. 2b) were used to determine the load substitute spectra.

a)

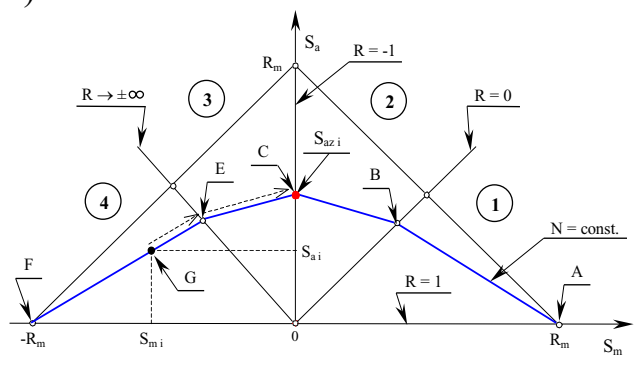

b)

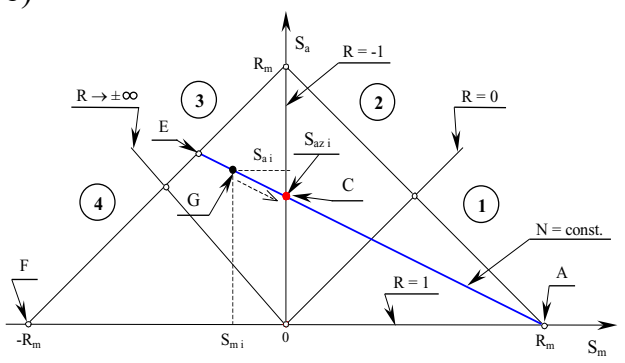

Fig. 2. Schematic presentation of the two-parameter characteristics models: a) Model IM, b) Model II. [2]

Model IM (Fig. 2a) was created on the basis of the model I presented in paper [4]. This model generalizes the Serensen's stress limit plot on the range of high cycle and low cycle fatigue.

Model IM (Fig. 2a) is divided into four zones, which are determined by required values of stress ratio $\mathrm{R}$. A line of constant fatigue life $(\mathrm{N}=$ const.) is plotted taking into account the stress amplitude Sai and the average value $S_{\mathrm{mi}}$ for sinusoidal cycle, and on this basis the determination of the equivalent amplitude $S_{a z i}$ is performed. Selection of stress amplitude value $S_{a i}$ on the ordinate axis, and mean value $S_{\text {mi }}$ on the abscissa axis determines the $G$ point. This point is interpolated to point $\mathrm{C}$, in which the line of constant fatigue life intersects with the ordinate axis, determining the load equivalent cycle with the average value $S_{m}=0$. Several load cycles, which are located on the same line of constant fatigue life, have the same equivalent stress amplitude $S_{\text {azi }}$ and are characterized by the stress ratio $\mathrm{R}=-1$ regardless of in which zone of two-parameter characteristic they are located.

In the model IM the line of constant fatigue life is described by the following formulas for four zones denoted from 1 to 4 (Fig.2b), which characterized by stress ratio variability. For zone 1 described by the variability of range of the stress ratio $0<\mathrm{R}<1$ the line of constant fatigue life is represented by the equation:

$$
N=N_{0}\left[\frac{S_{f(-1)} \cdot\left(S_{u}+S_{a(R)}^{(T)}-S_{m(R)}^{(T)}\right)}{S_{a(R)}^{(T)} \cdot S_{u} \cdot\left(1+\Psi_{N}\right)}\right]^{m_{(-1)}}
$$

The line of constant fatigue equation for zone 2 , in which the stress ratio $\mathrm{R}$ takes values -1 $\leq \mathrm{R} \leq 0$ is given in the form:

$$
N=\frac{N_{0} \cdot\left(S_{f(-1)}\right)^{m_{(-1)}}}{\left(S_{a(R)}^{(T)}+\Psi_{N} \cdot S_{m(R)}^{(T)}\right)^{m_{(-1)}}}
$$

The line of constant fatigue equation for zone 3 characterized by the variability of range of the stress ratio $-\infty<\mathrm{R}<-1$ is described by equation as follow:

$$
N=\frac{N_{0} \cdot\left(S_{f(-1)}\right)^{m_{(-1)}}}{\left(S_{a(R)}^{(T)}-\Psi_{N} \cdot S_{m(R)}^{(T)}\right)^{m_{(-1)}}}
$$

Zone 4 contains variability of cycle stress ratio $1<\mathrm{R}<+\infty$. In this zone the line of constant fatigue is determine by the equation: 


$$
N=N_{0}\left[\frac{S_{f(-1)} \cdot\left(S_{u}+S_{a(R)}^{(T)}+S_{m(R)}^{(T)}\right)}{S_{a(R)}^{(T)} \cdot S_{u} \cdot\left(1+\Psi_{N}\right)}\right]^{m_{(-1)}}
$$

Model II (Fig. 2b) for determining the equivalent load amplitude is a generalization of the Goodman's formula over the limiting fatigue life ranges low cycle and high cycle fatigue. This model is based on the Wöhler fatigue diagram for the stress ratio $R=-1$. Under load conditions characterized by stress ratio $\mathrm{R}=-1$, point $\mathrm{A}$ corresponds to the tensile strength of the material $S_{u}$, while point $C$ corresponds to the fatigue limit $S_{\mathrm{f}(-1)}$. The ACE line is the constant durability line $(\mathrm{N}=$ const.) For the sinusoidal load for the range of the cycle asymmetry coefficient $-\infty<\mathrm{R}<1$ with variable stress amplitudes Sai and mean values $\mathrm{S}_{\mathrm{mi}}$ is described by the following equation:

$$
N=N_{0}\left[\frac{S_{f(-1)}}{S_{a(R)}^{(T)}}\left(1-\frac{S_{m(R)}^{(T)}}{S_{u}}\right)\right]^{m_{(-1)}}
$$

On the basis of the described methods above of determination equivalent stress amplitudes, the block spectra of load for the applied load run were created. By means of the obtained sinusoidal cycles sets using the presented cycles counting methods, load spectra were developed.

\section{Results of fatigue life calculations (using a two-parameter fatigue characteristic)}

\subsection{Operational load}

In Figure 3 is presented run of stress changes in a structural element, which were recorded in operational load conditions. Due to use in calculations of fatigue life, run of stress changes is presented in the relative form $\mathrm{S}_{\mathrm{i}} / \mathrm{S}_{\max }$, where $\mathrm{S}_{\max }$ is the maximum value occurring in the load run.

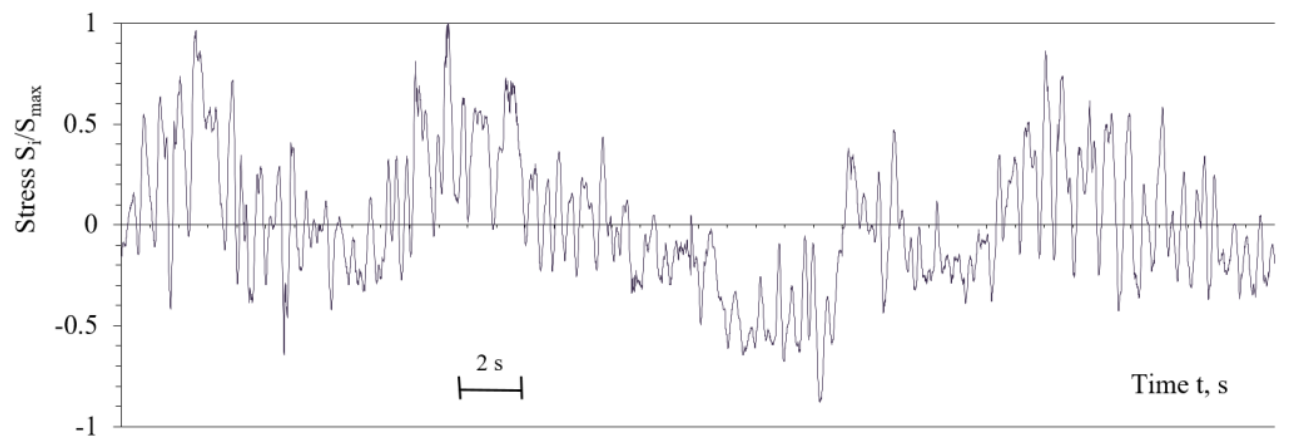

Fig. 3. Fragment of stress changes run in the form of the relative values $S_{i} / S_{\max }$.[2]

The load run presented in Figure 3 provide to determination of sinusoidal cycles set by use of the full cycle counting method. This method uses the assumption, that all ranges occurring in pairs within a definitive scope form cycles, that form closed hysteresis loops. This method schematization is based on progressive filtering of cycles with increasing amplitude value [5].

The obtained data sets contained sinusoidal cycles with $\mathrm{S}_{\mathrm{ai}} / \mathrm{S}_{\max }$ and $\mathrm{S}_{\mathrm{mi}} / \mathrm{S}_{\max }$ parameters from the variability of the stress ratio $-\infty<\mathrm{R}<1$, which is characteristic for broad spectrum loads. By means of determined data were appointed cycle set characterized by mean value $S m=0$ and equivalent amplitude $S_{a z}$ received by the use of two-parameter fatigue characteristic. Method of substitute amplitude $S_{a z}$ determination, described in point 2 , were applied. For assumed operational run determined block load spectra. The basis for 
the spectra development was the set of sinusoidal cycles determined by the adopted cycles counting methods. The S355J0 steel was used to perform calculations.

a)

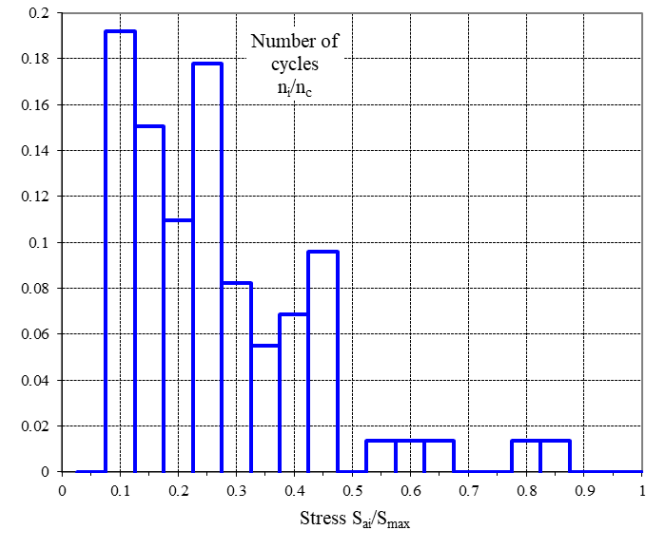

b)

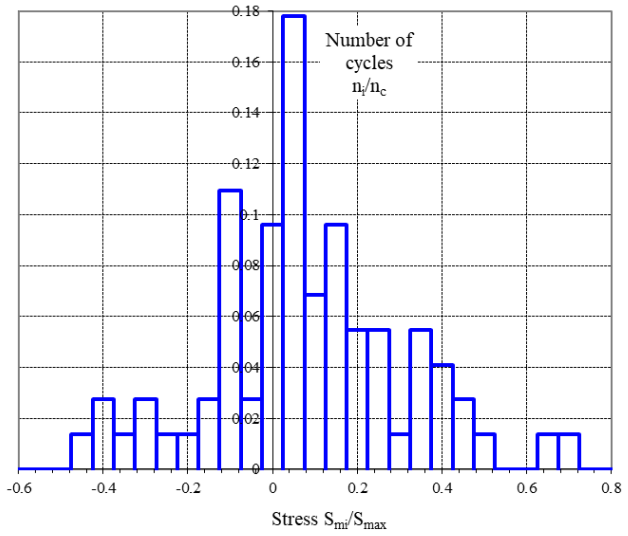

Fig. 4. Histograms of load cycles: a) for stress amplitude, b) for mean values.

The block load spectra (Fig. 5), used in fatigue life calculations, were determined for $\mathrm{S}_{\max }$ values $=500 \mathrm{MPa}$ for stress changes, taking into account model IM and model II of two-parameter fatigue characteristics. Figure 5a presents a block spectrum allowing the determined substitute amplitudes $\mathrm{S}_{\mathrm{az}}$ based on the IM model of a two-parameter fatigue characteristic. Whereas, Figure 5b illustrates a block spectrum determined by means on the II model of two-parameter fatigue characteristics.

a)

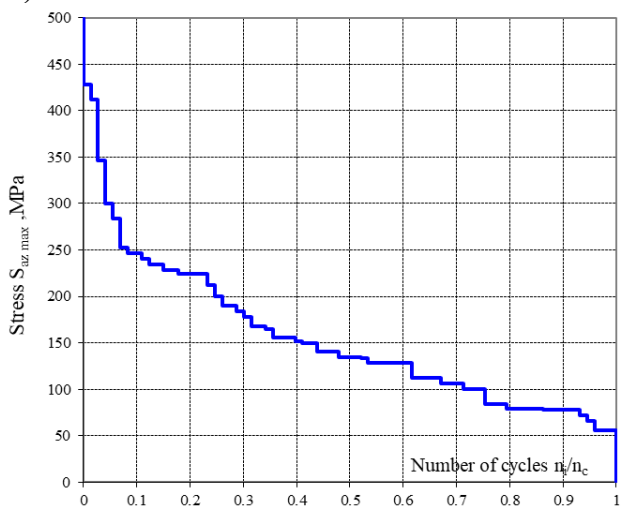

b)

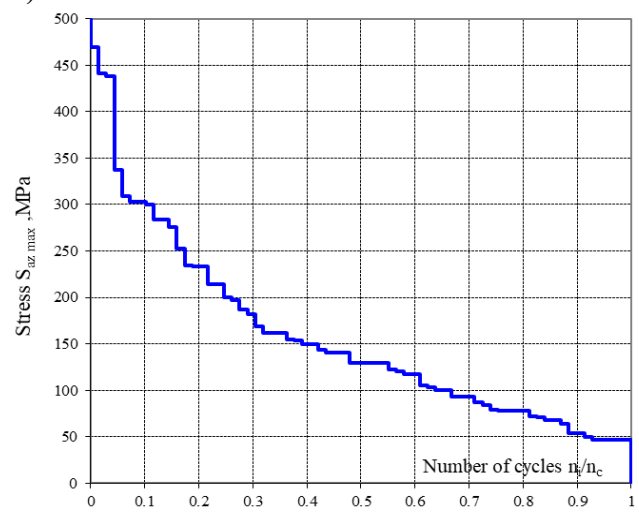

Fig. 5. Substitute block load spectra for $S_{\max }=500 \mathrm{MPa}$, determined for two-parameter fatigue characteristic: a) model IM, b) model II.

\subsection{Material properties}

The presented two-parameter fatigue characteristics were evolved for S355J0 steel. This material is a low-alloy steel with increased strength. Structure of the steel is composed of ferrite and pearlite grains. The S355J0 steel has the following static properties: tensile strength $\mathrm{Su}=678 \mathrm{MPa}$ and yield point $\mathrm{Sy}=499 \mathrm{MPa}$, and Young's modulus $\mathrm{E}=208159$ $\mathrm{MPa}$. Cyclic properties of S355J0 steel on the basis of the calculations were made: $\mathrm{m}_{(-1)}=$ $12,33, \mathrm{C}_{(-1)}=1.145 \cdot 10^{36}, \mathrm{~S}_{\mathrm{f}(-1)}=243.1 \mathrm{MPa}, \mathrm{k}=0.1386, \psi_{\mathrm{N}}=0,1205[5]$. 


\subsection{Calculations terms}

Fatigue life calculations was carried by means on the Palmgren-Miner's hypothesis on linear fatigue damage summation expressed as follows:

$$
\mathrm{D}=\sum_{\mathrm{i}=1}^{\mathrm{k}} \frac{\mathrm{n}_{\mathrm{i}}}{\mathrm{N}_{\mathrm{i}}}=1.0
$$

The fatigue life results were presented in the form of a number of accomplished load blocks, computed from the formula bellow:

$$
\lambda=\frac{1}{\mathrm{D}}
$$

By used of equivalent load spectra carried out the calculations of fatigue life. This calculations allowed to determine fatigue life diagram $\mathrm{Sa}(\mathrm{N})$. The fatigue life diagram compares the results of calculations for the model IM, model II of two-parameter fatigue characteristics and fatigue life experimentally determined. To calculate fatigue life, the Wöhler curve was determined experimentally under load conditions characterized by stress ratio $\mathrm{R}=-1$, described by the following formula:

$$
\log \mathrm{S}_{\mathrm{a}}=-\frac{1}{14,81} \log \mathrm{N}+3,0177
$$

On the basis of fatigue life calculation results for the IM model, a fatigue life chart is described by the equation:

$$
\log \mathrm{S}_{\mathrm{a}}=-\frac{1}{12,33} \log \mathrm{N}+3,1240
$$

For II model of the two-parameter fatigue characteristic, the fatigue life diagram is featured by the equation bellow:

$$
\log S_{a}=-\frac{1}{14,11} \log N+3,0283
$$

The results of fatigue life calculations complied in the form of the diagrams (Fig. 6). The chart presents calculations carrying out on the basis of substitute load spectra for the two-parameter fatigue characteristics with the results of experimental tests.

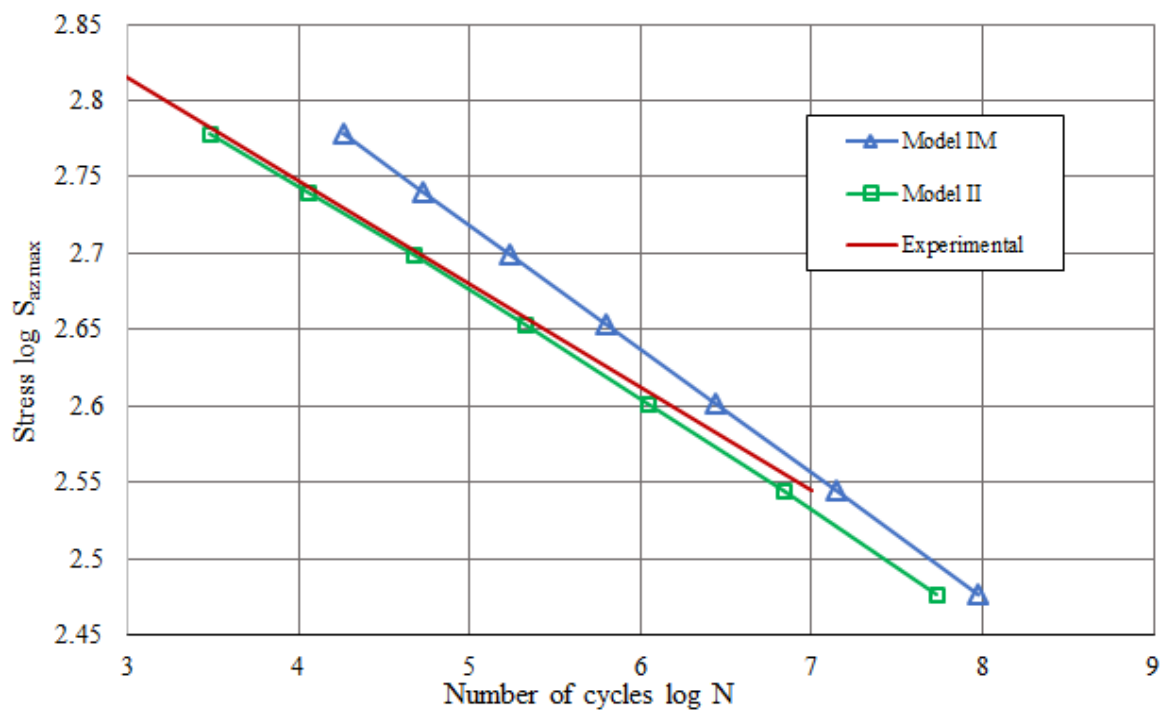

Fig. 6. Fatigue life diagram determined experimentally and for load spectra allowed two-parameter fatigue characteristics: model IM and model II. 


\section{Analysis of research results and summary}

The results of fatigue life calculations based on the adopted cycle counting method and selected two-parameter fatigue characteristics (model IM and model II) are characterized by discrepancy.

Due to calculations using two-parameter fatigue characteristics, the stress average value $\mathrm{Sm}$ is taken into account. As opposed to fatigue life calculations by means on Wohler diagram, which ignored the impact of mean value $\mathrm{Sm}$. This has a significant impact to assess the fatigue life of structural element. In particular, element that is exposed to board spectrum of operational loads.

On the fatigue life diagrams is presented that the highest durability is found in the results obtained on the basis of the substitute load spectra for the IM model of the twoparameter fatigue characteristic. The lowest fatigue life was obtained for calculations based on the model II of the two-parameter fatigue characteristic. Fatigue life for model II is similar to the durability determined during experimental tests. Meaning differences in the results of fatigue life calculations between model IM and model II of two-parameter characteristics may be affected the range of using chosen characteristics [3].

Model II is used for the stress ratio $-\infty<\mathrm{R}<-1$, ignored zone 4 (Fig.2) with range of stress ratio $1<\mathrm{R}<+\infty$. It can be said that the selection of the appropriate model of the two-parameter fatigue characteristic has a significant impact on the fatigue life calculations results. The choice of the proper model is related to the cyclic properties of researched material. .

In addition to the fatigue characteristics models, the cycle counting method also affects the fatigue life results. In cause that different cycle counting method is used, the calculated durability values would be different from each other.

\section{References}

1. Kocańda S., Szala J., PWN (2004)

2. Ligaj B., Sołtysiak R., Polish Meritime Research, no. 1 (2016)

3. Ligaj B., Szala G., Polish Meritime Research, no. 1 (2010)

4. Szala G., Ligaj B., Materials Science Forum, Fatigue Failure and Fracture Mechanics (2012)

5. Szala G., Ligaj B., Instytut Technologii Eksploatacji - Pańswtowego Instytutu Badawczego (2011) 